\title{
Can Hounsfield Unit Density Accurately Predict Recurrent Cholestatoma in Previously Operated Ears?
}

\author{
Egehan Salepci ${ }^{1}$, Muge Ugurlar ${ }^{1}$, Burcin Agridag Ucpinar ${ }^{2}$, Ali Gemalmaz ${ }^{2}$ and Suat Turgut ${ }^{1}$ \\ ${ }^{1}$ Department of Otorhinolaryngology, Sisli Hamidiye Etfal Research and Training Hospital, Turkey \\ ${ }^{2}$ Department of Radiology, Sisli Hamidiye Etfal Research and Training Hospital, Turkey
}

\begin{abstract}
The objective of the study was to determine whether Hounsfield Unit density would differentiate between cholesteatoma and other causes of opacification, such as granulation tissue in previously operated ears. Temporal bone computer tomography (CT) scans of 47 patients, who had revision mastoid surgeries, were evaluated retrospectively. A circular $5 \mathrm{~mm}^{2}$ region of interest (ROI) was placed at aditus ad antrum to measure Hounsfield Unit (HU) density. There was no statistically significant difference between cholesteatoma and non-cholesteatoma groups in terms of $\mathrm{HU}$ densities. Intraclass correlation coefficient test showed an intraclass correlation of 0.41 between measurements done by radiologists and otorhinolaryngologists, which showed poor reliability. Contrary to previous studies in unoperated ears, in operated ears HU density at aditus level cannot be a reliable diagnostic adjunct. Moreover, when it is considered that interrater reliability was poor between radiologists' and otolaryngologists' measurements, a need for further studies arise to solidify findings in this study.
\end{abstract}

Key Words: Cholesteatoma diagnostic imaging, Middle ear surgery, Revision mastoidectomy.

How to cite this article: Salepci E, Ugurlar M, Ucpinar BA, Gemalmaz A, Turgut S. Can Hounsfield Unit Density Accurately Predict Recurrent Cholestatoma in Previously Operated Ears?. J Coll Physicians Surg Pak 2021; 31(01):104-106.

Cholesteatoma is defined as the presence of keratinised squamous epithelium in the middle ear cavity. Although chronic otitis media without cholesteatoma can be amenable to medical treatment alone, presence of cholesteatoma necessitates surgical intervention. Recurrence of cholesteatoma after surgery is a common problem. Up to $70 \%$ recurrence rates of cholesteatoma intact canal wall and $17 \%$ for canal wall-down procedures have been reported. ${ }^{1}$

When considering a revision surgery, preoperative detection of cholesteatoma is crucial in treatment planning and surgical decision-making. Usefulness of computed tomography (CT) in detecting cholesteatoma decreases substantially in revision cases. Diffusion-weighted magnetic resonance imaging (MRI) improves the diagnostic rates of cholestatoma in previously operated ears. Even so, not all patients are able to tolerate the procedure and the costs are higherfor MRI scans than for $\mathrm{CT}^{2}{ }^{2}$

Hounsfield Unit (HU) density of CT is indicative of linear attenuation coefficient and every biological tissue has a distinct value between -1000 , which is that of air, and +1000 that is of compact bone.

Correspondence to: Dr. Egehan Salepci, Department of Otorhinolaryngology, Sisli Hamidiye Etfal Research and Training Hospital, Turkey

E-mail: egehansalepci@yahoo.com

Received: January 10, 2020; Revised: September 27, 2020; Accepted: October 28, 2020

DOI: https://doi.org/10.29271/jcpsp.2021.01.104
A recent study by Park et al. showed that a simple HU density measurement at aditus ad antrum would increase positive and negative predictive values of CT in detecting cholesteatoma, compared to routine evaluation of temporal bone CTs. ${ }^{3}$ In this study, the authors aimed to determine whether the same results would be found for revision cases and compared measurements made by radiologists and otolaryngologists. It was hypothesised that $\mathrm{HU}$ density measurements used by the otolaryngologist in an office-based setting, would be a simple adjunct to surgical decision-making for recurrent cholesteatoma cases.

Hospital discharge records of 69 patients, who had revision mastoid surgeries between April 2013 and March 2019 in Sisli Hamidiye Etfal Training and Research Hospital Otorhinolaryngology Clinic, were evaluated retrospectively. All patients were previously operated and had revision surgeries with suspicion of cholesteatoma. Of those 69 patients, four did not have preoperative CT so they were excluded from the study. Of the remaining 65,18 were excluded because they either had sclerotic mastoid cavities or no opacifications at aditus level, so HU densities could not be measured confidently. Consequently, a total of 47 patients were included in the study. Average age was 36.09 $( \pm 16.38)$ with youngest patient being of 10 and oldest 74 years.

The study was approved by the Sisli Hamidiye Etfal Research and Training Hospital Institutional Ethics Committee (Date 02.05.2019, No. 2260). The requirement of informed consent was waived by the Ethical Committee due to the retrospective study design. 
Preoperative non-enhanced temporal bone CT scan on a 128-section multidetector CT (MDCT) scanner (Somatom Definition AS Plus 128; Siemens, Forchheim, Germany) was performed to all patients included in the study within three months before the revision surgery. The parameters were 320 mA tube current, $120 \mathrm{kV} ; 128-0.625$ detector collimation; 0.5 second rotation time; $1 \mathrm{~mm} /$ rotation (pitch 0.391 ) table speed; $0.67 \mathrm{~mm}$ slice thickness; 4.9 seconds scan time; $96 \mathrm{~mm}$ field of view; and $512 \times 512$ matrix.

All MDCT scans were evaluated separately by a team of radiologists and otolaryngologists. Radiology team consisted of a Head and Neck Radiologist and a Radiology resident. Otolaryngology team consisted of an Otolaryngologist and an Otolaryngology resident. All examiners were blinded to the patients' clinical and post-surgical data. All measurements done on images were evaluated on Infinitt PACS Viewer Software (Infinitt North America, Phillipsburg, NJ).

To measure the average HU density, a circular region of interest (ROI) size of $5 \mathrm{~mm}^{2}$ was used because this was the largest size that could be used at aditus level in all of CT scans without interference by the adjacent bone. The anatomical reference points of the inner ears for antrum (lateral semi-circular canals) were lined symmetrically in the same plane for the optimal quantitative measurements. Aditus ad antrum was found on axial slices at the same level with the lateral semi-circular canal. The ROI circle was placed on aditus ad antrum and average HU density was obtained (Figure 1). Same measurements were done on one upper and one lower adjacent axial slice. Initial measurements at designated aditus level, smallest of the 3 measurements (aditus level, one upper and one lower slices) and mean of the three measurements were noted seperately (Tablel).

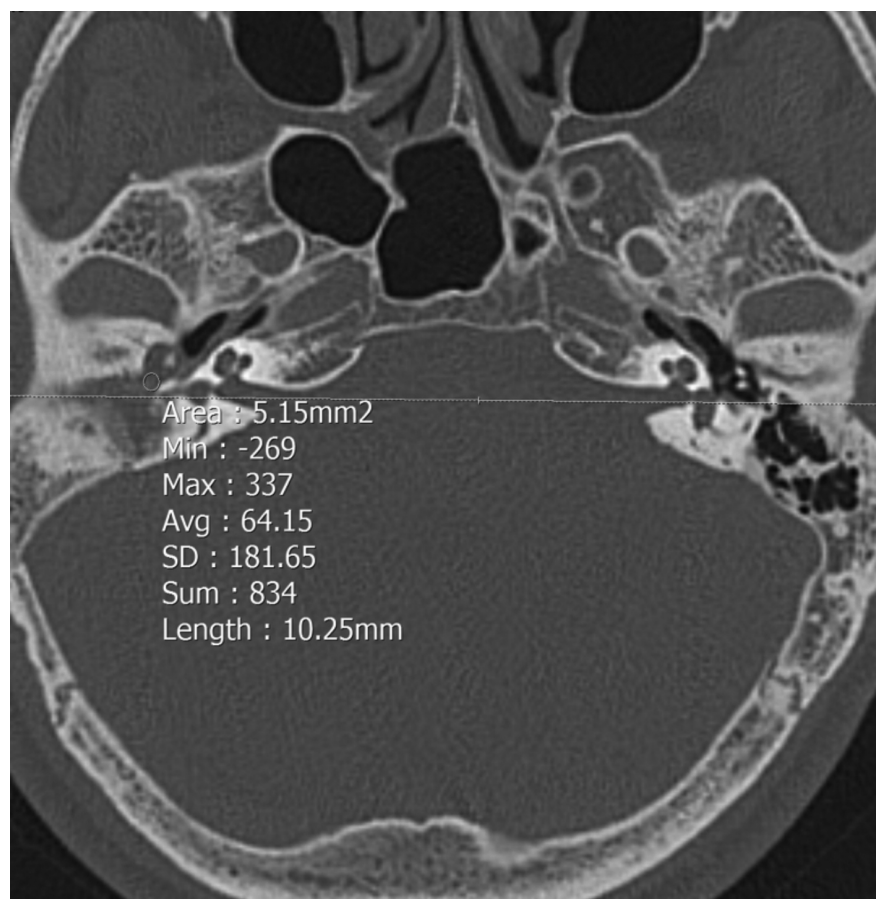

Figure 1: Measurement of mean HU density with ROI circle placed at aditus level in a patient with cholesteatoma.
Table I: Median and inter-quartile ranges of Hounsfield unit values of patients by cholesteatoma status as measured by otorhinolaryngologists and radiologists.

\begin{tabular}{|l|c|c|c|}
\hline & $\begin{array}{c}\text { Cholesteatoma } \\
\text { group median } \\
\text { (IQR) }\end{array}$ & $\begin{array}{c}\text { Non-cholesteatoma } \\
\text { group median } \\
\text { (IQR) }\end{array}$ & p-value \\
\hline Aditus ORL & $79(40.5)$ & $85(80)$ & 0.528 \\
\hline Aditus Rad & $76(40.75)$ & $74(49)$ & 0.707 \\
\hline Smallest ORL & $56.5(28)$ & $82(50)$ & 0.09 \\
\hline Smallest Rad & $56.5(32)$ & $52(49.5)$ & 0.65 \\
\hline Mean ORL & $79(33)$ & $98(56)$ & 0.219 \\
\hline Mean Rad & $83.5(38.5)$ & $76(42)$ & 0.859 \\
\hline
\end{tabular}

IQR: Inter-quartile range, ORL: Otorhinolaryngologist, Rad: Radiologist.

The $p$-values for cholesteatoma group vs. non-cholestatoma group.

Revision surgeries were all done in Sisli Hamidiye Etfal Training and Research Hospital between April 2013 and March 2019. All patients were operated because of recurrent purulent discharge from the ear; except for two tympanotomy patients, who were operated because of unexpected conductive hearing loss after the first surgery; and one canaloplasty patient, who had the surgery to correct external ear canal deformity present since the firstsurgery.

All patients were assessed about the presence of cholesteatoma during the surgery. Presence of squamous epithelium or keratin pearls in middle ear or mastoid cavity, was considered as compatible with cholesteatoma. If only inflammatory debris and/or granulation tissue were encountered and no trace of squamous epithelium was found in middle ear or mastoid cavity; the patient was deemed free of cholesteatoma. Cholesteatoma was found in 30 (63.83\%) patients, while 17 $(36.17 \%)$ patients were deemed cholesteatoma-free. During surgeries, no sign of tympanosclerosis was detected in any patients. No major complications were encountered after the surgeries and all patients were discharged within two days postoperatively.

For all statistical analyses, SPSS Version 20 (SPSS Inc, Chicago, IL) for Mac OS was used. While numerical data were expressed as mean \pm standard deviation when variables had normal distribution and as median (interquartile range) when variables had non-normal distribution, the categorical data were expressed as $n(\%)$. Shapiro Wilk's test was used to assess normality distributions. All variables were found not to be normally distributed, so Mann-Whitney U-tests of non-parametric were used to compare medians. A p value of less than 0.05 was considered as statistically significant. To determine interrater reliability of radiologists' and otolaryngologists' HU measurements, intraclass correlation coefficient test was conducted with two-way mixed model for absolute agreement.

When median HU values were compared between cholesteatoma and non-cholesteatoma groups, no statistically significant differences were found between the groups (Table I). When interrater reliability between measurements was assessed, intraclass correlation between radiologists' and otorhinolaryngologists' measurements at aditus level was found to be 0.41 , which showed poor reliability. 
Making the decision to operate on a previously operated ear with suspicion of cholesteatoma is a serious clinical problem. Detecting the presence of cholesteatoma in a patient undergoing revision surgery is important as it can help in planning the extent of surgery and patient counselling preoperatively. ${ }^{4}$

Although CT is a good diagnostic tool in detecting cholesteatoma in a patient with chronic otitis media, diagnostic value of CT decreases substantially for revision cases and differentiating between recurrent cholesteatoma, scar tissue and inflammatory fluid becomes virtually impossible. ${ }^{5}$

In this study, no statistically significant difference between medians of $\mathrm{HU}$ density values was found in cholesteatoma and non-cholesteatoma groups. Median values for first measured values and the smallest and the mean values of three measurements were compared. In none of these values, a statistically significant difference was found, but measured values were close to values reported in the study by Park et al. This discrepancy was caused by the previous drilling of the temporal bone because loss of bone tissue may result in a decreased $\mathrm{HU}$ density. ${ }^{3}$

Difficulty in identification of the level of the aditus because anatomy of the aditus, middle ear and mastoid cavity was disturbed by previous operations, may explain the poor interrater reliability. In unoperated ears, cholesteatoma usually starts at attic level and disease process includes aditus. However, residual or recurrent cholesteatoma may involve any part of the operated ear in which aditus level may be cholesteatoma-free, even there is recurrent disease. ${ }^{6}$ This also may explain high HU values measured at aditus level of some patients who had surgical findings compatible with cholesteatoma.

Lack of pathology result of every patient, small patient number, and study type being retrospective in design, are the limitations of this study.

\section{CONCLUSION}

This study showed that findings of the study by Park et al. could not be reproduced because of either specified limitations or HU densities at the aditus at antrum level are not significantly different between cholesteatoma in operated ears. Further studies designed prospectively, and with a larger patient group may clarify this issue.

\section{ETHICALAPPROVAL:}

The study was approved by the Sisli Hamidiye Etfal Research and Training Hospital Institutional Ethics Committee (Date 02.05.2019, No. 2260).

\section{PATIENTS' CONSENT:}

The requirement of informed consent was waived by the Ethical Committee due to the retrospective study design.

\section{CONFLICT OF INTEREST:}

The authors declared no conflict of interest.

\section{AUTHORS' CONTRIBUTION:}

ES: Contributed to design, data acquisition, statistical analyses and writing.

MU: Contributed to design, literature review and data acquisition.

BAU: Contributed to data analyses, literature review and writing.

AG: Contributed to data analyses, critical review and supervision.

ST: Contributed to critical review and supervision.

\section{REFERENCES}

1. Tomlin J, Chang D, Mc Cutcheon B, Harris J. Surgical technique and recurrence in cholesteatoma: A metaanalysis. Audiol Neurotol 2013; 18(3):135-42. doi: 10.1159/ 000346140.

2. De Foer B, Vercruysse JP, Bernaerts A, Deckers F, Pouillon M, Somers $\mathrm{T}$, et al. Detection of postoperative residual cholesteatoma with non-echo-planar diffusion-weighted magnetic resonance imaging. Otol Neurotol 2008; 29(4):513-7. doi: 10.1097/MAO.0b013e31816c7c3b.

3. Park MH, Rah YC, Kim YH, Kim JH. Usefulness of computed tomography Hounsfield unit density in preoperative detection of cholesteatoma in mastoid ad antrum. Am J Otolaryngol 2011; 32(3):194-7. doi.org/10.1016/j.amjoto. 2010.01.008

4. Berçin S, Kutluhan A, Bozdemir K, Yalçiner G, Sari N, Karameşe Ö. Results of revision mastoidectomy. Acta Otolaryngol 2009; 129(2):138-41. doi: 10.1080/000164808 02140893.

5. Tierney PA, Pracy P, Blaney SPA, Bowdler DA. An assessment of the value of the preoperative computed tomography scans prior to otoendoscopic "second look" in intact canal wall mastoid surgery. Clin Otolaryngol Allied Sci 1999; 24(4):274-6. doi: 10.1046/j.1365-2273.1999.00238.x.

6. Jackler RK. The surgical anatomy of cholesteatoma. Otolaryngol Clin North Am 1989; 22(5):883-96. www.ncbi. nlm.nih.gov/pubmed/2694067. 